Les vœux présidentiels au Portugal, en France et en Roumanie, et la crise internationale. Les enjeux des formes d'adresse et des procédés d'atténuation/ intensification

As mensagens presidenciais de fim do ano em Portugal, em França e na Roménia, e a crise internacional. A importância das formas de tratamento e dos processos de atenuação/intensificação

Presidential annual speeches in Portugal, France, Romania, and the international crisis. Address forms and means of mitigation/intensification Los mensajes presidenciales de fin de año en Portugal, Francia y Rumanía, y la crisis internacional. La importancia de las formas de tratamiento y de los procesos de atenuación/intensificación

\title{
Andreea Teletin
}

\section{OpenEdition}

Journals

Édition électronique

URL : https://journals.openedition.org/mots/21108

DOI : $10.4000 /$ mots. 21108

ISSN : 1960-6001

Éditeur

ENS Éditions

Édition imprimée

Date de publication : 22 avril 2013

Pagination : $31-46$

ISBN : 978-2-84788-393-0

ISSN : 0243-6450

Référence électronique

Andreea Teletin, «Les vœux présidentiels au Portugal, en France et en Roumanie, et la crise

internationale. Les enjeux des formes d'adresse et des procédés d'atténuation/intensification », Mots Les langages du politique [En ligne], 101 | 2013, mis en ligne le 22 avril 2015, consulté le 22 avril 2022. URL : http://journals.openedition.org/mots/21108; DOI : https://doi.org/10.4000/mots.21108 


\section{Les vœux présidentiels au Portugal, en France et en Roumanie, et la crise internationale. Les enjeux des formes d'adresse et des procédés d’atténuation/intensification}

Les allocutions de fin d'année des présidents - au Portugal, en France et en Roumanie - représentent une forme particulière de communication politique. Elles sont régies par des rituels plus ou moins stables et présentent un fort caractère démonstratif. Les présidents de la République construisent leur image notamment à travers les messages de vœux et, en même temps, font le bilan de l'année écoulée tout en éclairant les perspectives économiques et politiques à venir. Ce type singulier de discours renvoie à des énonciations orales qui impliquent un rapport orateur/auditoire ${ }^{1}$. Elles se rattachent donc au genre rhétorique des textes publiés après avoir été prononcés.

Partant de la crise internationale ${ }^{2}$ comme lieu commun des vœux présidentiels (2008-2010), nous étudierons la façon dont les formes nominales et pronominales d'adresse sont mobilisées pour s'adresser à autrui ou pour parler de soi-même et dont elles peuvent atténuer ou intensifier les actes de discours présents dans les allocutions de fin d'année de Cavaco Silva (CS), de Nicolas Sarkozy (NS) et de Traian Băsescu (TB).

Du point de vue théorique, notre étude tient ses fondements de la théorie sémantique de Bernard Pottier, qui considère le trimorphe comme le support universel des catégorisations de la pensée en tant qu'image mentale de

1. Les vœux présidentiels ont fait l'objet de plusieurs travaux en France, alors qu'au Portugal et en Roumanie, il s'agit d'un domaine à explorer. Mentionnons quelques références sur les vœux présidentiels français : à propos des positionnements énonciatifs, Leblanc et Martinez (2005); du je présidentiel, Leblanc et Fiala (2004); de l'ethos, Leblanc (2003). Pour une analyse comparative des vœux des présidents Traian Băsescu et Nicolas Sarkozy d'un point de vue discursif et sémiotique, voir l'article de Marina Ciolac (2010); pour celle des présidents sous la Cinquième République, voir la thèse de doctorat de Jean-Marc Leblanc (2005).

2. La crise économique d'échelle internationale de l'automne 2008 a débuté au cours de l'été 2007 avec la crise des subprimes aux États-Unis et a atteint rapidement la plupart des pays, y compris ceux de l'Union européenne.

Fundação para a Ciência e a Tecnologia / Centre de linguistique de l’Université de Porto andreeateletin@hotmail.com 
la représentation. Le trimorphe est une représentation triphasée d'un événement ou d'une catégorisation; à travers un schéma mental, on visualise de la manière la plus abstraite possible les cinétismes et les dynamismes constituant un événement et reflétant au plus près l'image qu'on suppose avoir à l'esprit (Pottier, 1992).

De cette manière, l'approche trimorphique de l'événement «crise», selon les trois phases du trimorphe (approche, contact, éloignement), nous permettra de mettre en relation les discours des trois présidents et d'étudier des séquences textuelles plus ou moins étendues où ce lexème apparaît.

\section{Objectifs et repères théoriques}

En nous inspirant des travaux de Bernard Pottier, nous combinerons les perspectives sémasiologique et onomasiologique. D'un point de vue onomasiologique, nous avons délimité deux catégories sémantiques : la désignation (de l'autre et de soi-même) et la modalité (selon l'appareil conceptuel proposé par Pottier).

Pour ce qui est de la désignation, quelle que soit la langue, le système des formes d'adresse comprend des formes allocutives, des formes élocutives et des formes délocutives.

Les formes d'adresse jouent un rôle déterminant dans ce type d'interaction, car elles constituent une ressource dont disposent les présidents pour construire la relation interpersonnelle avec leurs interlocuteurs. De plus, elles peuvent se charger de valeurs particulières en fonction de l'acte de langage auquel elles se trouvent associées. Elles contribuent ainsi à adoucir ou à renforcer la valeur illocutoire de ces actes de langage. Pour mieux appréhender leurs atouts adoucissants et intensificateurs, nous étudierons de quelle façon les formes d'adresse fonctionnent comme des guides d'interprétation de la crise internationale partagée par les trois pays à l'étude. Du point de vue de la politesse (Araújo Carreira, 1997, Kerbrat-Orecchini, 1990), et compte tenu du corpus examiné, il s'avère pertinent de postuler que les actes menaçants sont atténués alors que les actes valorisants sont renforcés.

Pour ce qui est de l'étude des formes d'adresse dans les trois langues romanes concernées «en tant que supports linguistiques de la désignation de l'autre, mais aussi de soi-même «3 (Araújo Carreira, 2008, p.11), elles seront envisagées sur le plan des modalités énonciatives. En nous appuyant également sur les travaux de Catherine Détrie (2008) à propos de l'apostrophe nominale4 et de Ruth Amossy (2000) à propos de l'ethos discursif, nous analyserons

3. Voir, à propos des termes d'adresse et des modalités énonciatives dans les langues romanes, Araújo Carreira éd. (2008).

4. Dans cette étude, Catherine Détrie (2006, p. 20) considère l'apostrophe nominale comme 
les enjeux discursifs des formes nominales et pronominales d'adresse, et nous nous intéresserons à la construction d'identités individuelles et collectives présentes dans les vœux des trois présidents. Nous verrons ainsi les stratégies discursives qui contribuent à la construction de l'ethos du locuteur dans les vœux présidentiels, notamment la dimension argumentative des formes d'adresse et la manière dont les présidents orientent leurs discours pour construire des images d'eux-mêmes et du public auquel ils s'adressent.

La modalité sera envisagée ici au sens large, comme l'entend Bernard Pottier (1992, p. 204-206), c'est-à-dire comme une catégorie sémantique, avec ses quatre zones modales : la modalité existentielle (ontique et aléthique) - «une généralisation qui se veut indépendante du JE, donc universelle, prendra la forme de la modalité existentielle (ontique et aléthique) », la modalité épistémique - « orientée vers la perception, le savoir» -, la modalité factuelle ou déontique - «orientée vers le faire ou le dire» - et la modalité axiologique - «[...] le jugement porté sur tout ce qui est formulé», ce qui nous permettra d'analyser la variété des manifestations énonciatives des trois présidents de la République. Ces allocutions à caractère épidictique ont comme finalité de convaincre les auditeurs, à l'heure du bilan, du succès des décisions économiques prises et des politiques menées : il s'agit d'un discours qui subit une modalisation déontique ou factuelle (agir sur l'autre), laquelle se combine à son tour avec les modalités épistémique (les degrés de connaissance) et axiologique (les valeurs). C'est pourquoi notre étude se centre sur ces trois types de modalité. Tout comme le souligne Maria Helena Araújo Carreira (1997, p. 231-232), «la théorie de Bernard Pottier donne une place primordiale au "domaine modal" tout en l'articulant avec les trois autres domaines conceptuels: Temps, Espace, Notion ». Les catégories modalité et personne se trouvent en relation étroite, comme le montrent très clairement les citations suivantes : «Le JE énonciateur est le maître des modalités» et «Tout propos est susceptible d'être modalisé parl'énonciateur» (Pottier, 1992, p. 204).

\section{Les vœux présidentiels, un genre spécial}

Selon Leblanc et Martinez (2005), « les messages de vœux des présidents de la République constituent un genre discursif particulier, lié à l'épidictique, et fournissent un angle d'approche original pour l'analyse de l'ethos présidentiel vu du rituel politique».

Du point de vue discursif, les vœux présidentiels représentent dans les trois pays une forme de discours légitime, à visée cérémonielle. D'autres éléments

une forme d'adresse, dont la fonction est de «modeler par une forme langagière le tu auquel s'adresse le je». 
communs concernent l'orientation politique des trois présidents (la droite) et le type de communication verbale envisagée - orale, unilatérale et télédiffusée.

Cependant, les conditions socioculturelles dans les trois pays sont différentes : le contexte situationnel de la communication est très formel pour les vœux du président Nicolas Sarkozy et du président Cavaco Silva qui prononcent leurs discours à partir du palais de l'Élysée, à Paris et du palais de Belém à Lisbonne, seuls devant les caméras. De plus, au Portugal et en France, il existe une longue tradition des vœux présidentiels (même endroit de la transmission, même façon de transmettre les vœux). En Roumanie, pendant la dictature de Nicolae Ceauşescu, les vœux présidentiels étaient devenus une corvée nationale puisqu'ils étaient longs et mensongers. C'est pourquoi on retrouve des stratégies de communication différentes des présidents roumains après la révolution de 1989 : tous ont essayé d'innover sur le plan de la forme et du fond pour éviter toute ressemblance avec les vœux de l'ancien dictateur. Ainsi, le président roumain prononce ses discours sur une scène, à l'extérieur, au milieu des gens venus fêter le Nouvel An. Traian Băsescu n'est jamais seul devant les caméras : à ses côtés se trouvent ou des politiques locaux, ou sa famille. Les destinataires du président français et du président portugais sont uniquement les téléspectateurs, au contraire des destinataires du président roumain qui sont de deux types : les téléspectateurs et l'auditoire local. Ces distinctions concernant les conditions externes ont une répercussion sur les différentes façons de présenter les vœux - façon sobre (rythme lent de la lecture, prononciation claire, gestes pondérés) pour Nicolas Sarkozy et Cavaco Silva, face au comportement communicatif très familier et détendu de Traian Băsescu.

\section{Analyse comparative des vœux présidentiels}

Pour notre étude, nous avons utilisé les transcriptions des allocutions, disponibles sur les sites internet officiels ${ }^{5}$. Nous examinerons ici l'élément perturbateur (la crise) et la manière dont les présidents gèrent au niveau discursif les supports linguistiques de la désignation de l'autre et de soi-même à travers les séquences d'ouverture, centrales et de clôture des allocutions dans les trois pays à l'étude.

Les séquences d'ouverture sont très courtes dans les discours de Nicolas Sarkozy et de Cavaco Silva. Ainsi, dans le cas des discours du président portugais, elles contiennent uniquement la formule de salut : "Boa noite!» («Bonsoir!»)6. Pour ce qui est du discours de Nicolas Sarkozy, il recourt à la formule

5. Corpus : http://www.presidencia.pt, www.elysee.fr, www.presidency.ro.

6. Toutes les traductions des exemples en portugais et en roumain ont été faites par nos soins à partir des vœux présidentiels disponibles sur Internet, sur les sites officiels de la présidence de chaque pays. 
d'adresse «Mes chers compatriotes» en décembre 2008. Dans les vœux de décembre 2009, cette formule est précédée par la forme allocutive orthonymique très courte : «Françaises, Français! Mes chers compatriotes ! En ce qui concerne les discours du président roumain, les deux premières séquences d'ouverture sont plus longues, compte tenu de la double nature de son public. Dans les vœux pour 2009, qui sont prononcés à Braşov, la séquence initiale est constituée de la formule de salut «Bună seara» ("Bonsoir»), ainsi que des vœux de bonne année adressés au public local : «La mulţi ani braşoveni!» («Bonne année, habitants de Braşov!») et des vœux adressés à toute la nation : «La mulţi ani, români!» («Bonne année, Roumains!») Dans les vœux pour 2010, la gamme des destinataires du président s’élargit beaucoup plus. Traian B־asescu commence par souhaiter une bonne année à tous les Roumains (forme d'adresse collective) puis énumère les concitoyens de plusieurs ethnies, pour finir par des vœux adressés à l’armée :

TB : La mulţi ani, români ! La mulţi ani, români din interiorul frontierelor şi din afara frontierelor României! La mulţi ani, cetăţeni români, etnici romi, maghiari, ucraineni, tătari, turci, sârbi şi de orice altă naţionalitate sub cetăţenie română! La mulţi ani, Armatei Române, fie ea acasă sau în teatrele de operaţii din Afganistan sau din Balcanii de Vest!7

Pour ce qui est des séquences centrales, elles commencent toutes par une caractérisation de l'année achevée. Si le trimorphe de Bernard Pottier présente trois mouvements (approche, contact, éloignement), la même optique peut s'appliquer à la crise en tant qu'événement (naissance, évolution, fin espérée). La crise financière et économique amorcée en 2007 a connu une brusque accélération en 2008. D'ailleurs, comme nous allons le constater, les discours des présidents portugais et français conçoivent l'événement en trois mouvements. Une première approche de la crise qui est « rude » pour Nicolas Sarkozy et «difficile» pour Cavaco Silva, selon les exemples ci-dessous :

NS : L'année 2008 s'achève. Elle a été rude. C'est la raison pour laquelle je veux penser d'abord à ceux que la vie a durement éprouvés, à ceux qui ont perdu leur emploi sans y être pour quoi que ce soit, à ceux qui sont victimes d'injustice, à ceux qui doivent affronter l'absence d'un être cher. Je veux penser à nos soldats qui en ce moment même risquent leur vie pour notre sécurité et pour la paix. Je veux penser à leurs familles qui vivent douloureusement cette séparation. Et plus encore à ceux qui pleurent un fils, un mari, un fiancé, un père. Pour tous les Français, cette année a été difficile.

7. «Bonne année Roumains! Bonne année, Roumains de l'intérieur et de l'extérieur des frontières de la Roumanie! Bonne année, citoyens roumains, d'ethnie tsigane, hongroise, ukrainienne, tatare, turque, serbe et de toute autre nationalité sous citoyenneté roumaine! Bonne année à l'armée roumaine, qu'elle soit chez elle ou dans les théâtres d'opérations de l'Afghanistan ou des Balkans de l’ouest!». 
CS : Quero começar por dirigir uma palavra especial de solidariedade a todos os que se encontram em situações particularmente dificeis porque sofreram uma redução inesperada dos seus rendimentos. 8

Les présidents portugais et français s'adressent à des allocutaires collectifs au sein de différents groupes. Le président Sarkozy mentionne des destinataires malheureux, les chômeurs, les soldats, les familles des soldats, les victimes d'injustice, en attirant ainsi l'attention sur la difficulté de l'année 2008. Nous avons une vision dysphorique de la crise, marquée linguistiquement par l'emploi d'adverbes et d'adjectifs qualificatifs se reportant à un passé récent (l'année qui vient de s'écouler). D'ailleurs, les termes et expressions difficile, rude, difficultés, faire des efforts reviennent dans son discours assez régulièrement, tout comme les différentes relations de qualification de la notion de crise, financière d'abord, économique ensuite. Le lexème crise apparaît également dans des syntagmes qualificatifs hyperboliques, par exemple : « une période de crise comme le monde n'en avait pas connu depuis bien longtemps». Cavaco Silva, gardant le même ton sobre que le président français et évoquant les mêmes difficultés, construit son discours en interpellant toutes les personnes en situation difficile, pour ensuite s'adresser à trois catégories de gens en particulier dans son discours - les jeunes, les agriculteurs et les petits commerçants :

$\mathrm{CS}:$ A estes homens e a estas mulheres, que sofrem em silêncio, e que até há pouco tempo nem sequer imaginavam poder vir a encontrar-se na situação que agora atravessam, quero dizer-lhes, muito simplesmente : não se deixem abater pelo desânimo. O mesmo digo aos jovens que, tendo terminado os seus estudos, vivem a angústia de não conseguirem um primeiro emprego : acreditem nas vossas capacidades, não percam a vontade de vencer. Quero também lembrar dois outros grupos da nossa sociedade que são frequentemente esquecidos e que vivem tempos difíceis. Os pequenos comerciantes, que travam uma luta diária pela sobrevivência. o pequeno comércio deve merecer uma atenção especial porque constitui a única base de rendimento de muitas famílias. Os agricultores, aqueles que trabalham a terra, que enfrentam a subida do preço dos adubos, das rações e de outros factores de produção. 9

8. «Je veux commencer par un mot spécial de soutien pour tous ceux qui se trouvent dans des situations particulièrement difficiles parce qu'ils ont subi une réduction inattendue de leurs revenus. »

9. «Àvous, hommes et femmes qui souffrez en silence et qui, jusqu'à très récemment, n'imaginiez même pas que vous pourriez vous retrouver dans une situation pareille, je veux dire très simplement : ne vous laissez pas vaincre par le découragement. Je dis la même chose aux jeunes qui, ayant terminé leurs études, vivent dans l'angoisse de ne pas obtenir un premier emploi : croyez en vos capacités, ne perdez pas la volonté de gagner. Je veux également évoquer deux autres groupes de notre société, qui sont souvent oubliés et qui vivent des moments difficiles. Les petits commerçants, qui sont engagés dans une lutte quotidienne pour la survie. Le petit commerce doit mériter une attention particulière car il constitue l'unique source de revenus pour de nombreuses familles. Les agriculteurs, les personnes qui travaillent la terre et qui font face à la hausse des prix des engrais, des aliments et d'autres facteurs de production. » 
Nous attirons l'attention sur la construction en miroir de ces discours : la thématique des vœux qui constitue l'aspect central des messages, et le champ lexical de la souffrance qui apparaît dans les deux cas. L'ethos repose ainsi sur un discours à la fois dysphorique et compassionnel des présidents qui souffrent avec leur peuple. À l'autre bout de l'Europe et du pôle émotif, les propos du président roumain sont modalisés positivement d'un point de vue axiologique car il utilise un lexique euphorique lié à la fête :

TB : Se-ncheie un 2008 care-a fost un an bun pentru România. Vreau să vă asigur aici, la Braşov, pe dumneavoastră, pe braşoveni, pe toţi românii că instituţiile statului român au şi resurse, au şi capacitatea să facă ca anul 2009 să fie un an în care să trăim mai bine. ${ }^{10}$

Les vœux adressés aux Roumains où apparaissent de façon claire les interlocuteurs (la forme pronominale «vous», les syntagmes nominaux «les habitants de Braşov», «tous les Roumains »), les marqueurs spatio-temporels («ici, à Braşov») et les termes axiologiques positifs («bonne année », «vivre mieux») construisent l'image d'un président garant du bonheur de son peuple. Cependant, le discours de Traian Băsescu est «délibérément trompeur», selon les termes de Mariana Ciolac (2010):

Même si l'on peut considérer que les effets de la crise se sont propagés moins vite vers les frontières de l'UE, l'appréciation de T. Băsescu est plutôt subjective, sinon délibérément trompeuse, dans la tradition des discours totalitaires, où l'on faisait l'apologie d'un niveau de vie présenté comme élevé, quand en réalité il était complètement défaillant. De plus, le président roumain minimise les effets et les conséquences à venir de la crise économique mondiale. Montrant un optimisme exagéré, il recommande même une confiance sans justification réelle dans les institutions de l'État roumain, capables, selon lui, de conduire les Roumains en 2009 vers une vie meilleure.

D'ailleurs, le président roumain ne mentionne dans son discours qu'une catégorie restreinte de destinataires, tous des électeurs potentiels pour l'année 2009: les Roumains travaillant à l'étranger, les militaires roumains en opération et les jeunes. Il s'agit de l'électorat à l'aide duquel il a gagné, d'un léger écart, les élections présidentielles face à son concurrent socialiste :

TB : De aici, din centrul ţării, vreau să ne amintim acum de milioanele de români care lucrează în Italia, în Spania, în toată Europa, în toată lumea, vreau să ne gândim acum la militarii români din Afganistan, din Irak, din Balcani, din Africa, care îşi servesc ţara oriunde s-ar afla în lume. Şi, înainte de a trece la Anul Nou, aş vrea să mă adresez în mod special tinerilor : avem nevoie de implicarea generaţiei tinere în

10. «L'année 2008 qui s’achève a été une bonne année pour la Roumanie. Je veux vous assurer ici à Brasov, vous les habitants de Brasov, et vous, tous les Roumains, que les institutions de l’État roumain ont les ressources aussi bien que la capacité de faire en sorte que l'année 2009 soit une année pendant laquelle nous puissions vivre mieux.» 
viaţa politică, în viaţa economică, în viaţa socială. Am convingerea că schimbarea rapidă a României poate fi făcută numai împreună cu generaţia tânără. ${ }^{11}$

Une fois construite la représentation de l'allocutaire, déterminé par un ensemble de qualifications qui, finalement, n'est pas très différent dans les trois pays à l'étude (les jeunes, les militaires, les chômeurs, etc.), c'est le tour des présidents, à la première personne, de s'autoqualifier face à la crise :

CS : Portugueses, não devo esconder que 2009 vai ser um ano muito difícil. Receio o agravamento do desemprego e o aumento do risco de pobreza e exclusão social. Devo falarverdade. A verdade é essencial para a existência de um clima de confiança entre os cidadãos e os governantes. É sabendo a verdade, e não com ilusões, que os portugueses podem ser mobilizados para enfrentar as exigências que o futuro lhes coloca. ${ }^{12}$

NS : Face à cette crise je mesure la responsabilité qui est la mienne. Cette responsabilité, je l'assumerai pour que tous ceux qui en ont besoin soient protégés par l'État et que notre pays sorte plus fort de cette épreuve. Depuis que les difficultés sont apparues je vous ai toujours dit la vérité et j'ai agi. C'était mon devoir.

Dans la représentation trimorphique de la zone de contact, nous observons une focalisation sur la désignation élocutive je avec pour caractérisation des qualités présidentielles dire la vérité, ce qui est présenté comme essentiel par Nicolas Sarkozy et Cavaco Silva. Cependant, la construction de l'image du président français et de son allocutaire repose sur des supports linguistiques différents par rapport à l'image du président portugais et à la manière dont il conçoit son public. Nicolas Sarkozy privilégie les marques de première personne du singulier (23 occurrences du je présidentiel) tout en utilisant des verbes d'action («j'ai fait», «j'ai essayé de changer l'Europe») et des verbes volitifs, ce qui crée un processus d'intensification de ses actes et gestes. Cela lui permet de s'engager, mais aussi de défendre ses choix et ses positions. De plus, la présence massive des verbes épistémiques intensifie l'expression de la modalisation déontique qui suit: «je sais », «j'ai la conviction que l’Europe ne doit pas subir mais agir et protéger», «Je pense enfin à la réforme de notre procédure pénale si importante pour mieux protéger nos libertés individuelles».

11. «D’ici, du centre du pays, je veux qu'on se souvienne maintenant des millions de Roumains qui travaillent en Italie, en Espagne, dans toute l'Europe, partout dans le monde, je veux qu'on pense maintenant aux soldats roumains en Afghanistan, en Irak, dans les Balkans, en Afrique, qui servent leur pays où qu'ils soient dans le monde. Et, avant le passage au Nouvel An, je voudrais m’adresser plus spécialement aux jeunes : nous avons besoin de l'implication de la jeune génération dans la vie politique, dans la vie économique, dans la vie sociale. J'ai la conviction que le changement rapide de la Roumanie ne peut être entrepris qu'ensemble avec la jeune génération. »

12. «Portugais, je ne dois pas cacher que 2009 sera une année très difficile. Je crains l'aggravation du chômage et le risque accru de pauvreté et d'exclusion sociale. Je dois dire la vérité. La vérité est essentielle pour l'existence d'un climat de confiance entre les citoyens et les gouvernants. C'est en sachant la vérité, et non pas avec des illusions, que les Portugais peuvent être mobilisés pour répondre aux exigences que le futur leur prépare.» 
Nous assistons à un véritable jeu de formes d'adresse (voir, à propos de l'alternance des pronoms personnels dans les discours politiques, l'étude de Araújo Carreira, 2005). Ainsi, du point de vue du schéma trimorphique de la crise, l'agencement des formes d'adresse part du vous-je - «Depuis que les difficultés sont apparues je vous ai toujours dit la vérité et j'ai agi. C'était mon devoir »- passe par le nous-je pour parler de la crise et des issues possibles "Les difficultés qui nous attendent en 2009 seront grandes. J'en suis pleinement conscient. Je suis plus décidé que jamais à y faire face, avec le souci de la justice, avec l'obsession d'obtenir des résultats. Nous serons pragmatiques, attentifs, réactifs et s'il faut faire davantage, nous le ferons mais en gardant notre sang-froid. Les difficultés, mes Chers Compatriotes, nous avons les moyens de les affronter» - et finit par je-vous : "La crise nous oblige à changer plus vite et plus profondément. La crise est une épreuve. Elle est aussi un défi. Ce défi-là, je veux le relever avec vous. Vous pouvez compter sur moi.» Comme nous pouvons le voir, il s'agit d'une forte personnalisation du discours qui souligne le rôle de Nicolas Sarkozy en tant que président de la République.

Quant au discours de Cavaco Silva, c'est un processus d'atténuation de la prise en charge énonciative, grâce à l'emploi des structures délocutives, qui se dégage. Ainsi, si au début on assiste à une prise de position par le je face à la crise dans la zone d'approche, les énoncés suivants prennent la forme d'une séquence descriptive, riche en constats, parsemée d'occurrences du devoir :

CS : A crise chegou quando Portugal regista oito anos consecutivos de afastamento em relação ao desenvolvimento médio dos seus parceiros europeus. Há uma verdade que deve ser dita: Portugal gasta em cada ano muito mais do que aquilo que produz. Portugal não pode continuar, durante muito mais tempo, a endividar-se no estrangeiro ao ritmo dos últimos anos. Para quem ainda tivesse dúvidas, a crise financeira encarregou-se de desfazê-las. Como é sabido, quando a possibilidade de endividamento de um País se esgota, só resta a venda dos bens e das empresas nacionais aos estrangeiros. Os portugueses devem também estar conscientes de que dependemos muito das relações económicas com o exterior. ${ }^{13}$

Le discours de Cavaco Silva se caractérise donc par une prise de distance énonciative produite par le rejet des marques de la première personne du singulier et de la deuxième personne du pluriel, la prise en charge de l'énoncé étant assurée par les référents - les Portugais et le Portugal. Cependant, le je

13. «La crise est survenue alors que le Portugal enregistre depuis huit ans une certaine distance par rapport au développement moyen de ses partenaires européens. Il y a une vérité qui doit être dite : le Portugal dépense chaque année beaucoup plus qu'il ne produit. Le Portugal ne peut continuer plus longtemps à emprunter à l'étranger au rythme des dernières années. Pour ceux qui auraient encore eu des doutes, la crise financière s'est chargée de les détruire. Comme on le sait, lorsque la possibilité de l'endettement d'un pays est épuisée, il ne reste que la vente des biens et des entreprises nationales aux étrangers. Les Portugais doivent aussi être conscients que nous dépendons beaucoup des relations économiques avec le monde extérieur. » 
est une sorte de médiateur qui se met du côté de la nation et dont le discours est marqué à la fois par les modalités factuelle et axiologique :

CS : Portugueses, já passámos por outras situações bem difíceis. Não nos resignámos e fomos capazes de vencer. O mesmo vai acontecer agora. Tenho esperança e digo-o com sinceridade. ${ }^{14}$

Les séquences centrales des vœux présidentiels de 2009-2010 méritent également une attention particulière car les conséquences de la crise économique mondiale ne peuvent plus être ignorées. De plus, les bilans des présidents se reflètent dans leurs vœux, et leurs allocutions se distinguent par une profusion des valeurs, verbes et notions fondatrices qui convergent vers un même effet - notamment un discours tourné vers l'action. Mentionnons que chacun de ces trois discours introduit un concept clé : la fraternité dans les vœux de Nicolas Sarkozy, les valeurs dans ceux de Cavaco Silva, la solidarité dans ceux de Traian Băsescu.

NS : « Face à l'isolement, face à la solitude, si répandus dans nos sociétés modernes, je souhaite que 2010 soit l'année où nous redonnerons un sens au beau mot de fraternité qui est inscrit dans notre devise républicaine.»

CS : Há que recuperar o valor da família. O esbatimento dos laços familiares tem sido um dos factores que mais contribuem para agravar as dificuldades que muitos atravessam. Devemos também valorizar a prática do valor da ética republicana. [...]. Temos também de restaurar o valor da confiança nas instituições e na justiça. Os Portugueses têm de acreditar que existe justiça no seu País, que ninguém está acima da lei. 15

TB : Avem nevoie ca, în perspectiva anilor viitori, să facem din solidaritate un mod de a trăi. Românii trebuie să fie solidari cu aspiraţiile lor, trebuie să fie solidari cu ei şi, toţi la un loc, solidari cu ţara. ${ }^{16}$

Comme nous allons le voir à présent, la crise sert de topos pour introduire des séquences discursives qui ont pour but principal de dresser le bilan des actions politiques, économiques et sociales des trois présidents. Nous notons, en particulier, un suremploi de la première personne du pluriel par rapport aux discours de l'année précédente. En effet, leurs discours sont marqués par un

14. «Portugais, nous sommes déjà passés par d'autres situations très difficiles. Nous ne nous sommes pas résignés et nous avons été capables de triompher. La même chose va se produire maintenant. J'ai de l'espoir et je le dis avec sincérité.»

15. «Il faut restaurer la valeur de la famille. La disparition des liens familiaux est l'un des facteurs qui contribuent le plus à aggraver les difficultés que beaucoup traversent. Nous devons valoriser également la pratique de l'éthique républicaine. Nous devons aussi restaurer la valeur de la confiance dans les institutions et la justice. Il faut que les Portugais croient qu'il existe une justice dans leur pays, que personne n'est au-dessus de la loi. »

16. «Nous avons besoin, dans la perspective des années à venir, de faire de la solidarité un mode de vie. Les Roumains doivent être solidaires de leurs aspirations, solidaires d'eux-mêmes et, tous ensemble, solidaires du pays.» 
nous de solidarité avec les destinataires. Cette idée est exprimée par les mêmes processus énonciatifs dans les trois discours présidentiels. En voilà quelques exemples: le pronom personnel nous, les déterminants possessifs notre, nos, les adjectifs unis, solidaires, rassemblés, l'adverbe ensemble. Si l'on rajoute à l'emploi explicite de ce nous la présence implicite du pronom dans la désinence des verbes à l'impératif, nous constatons des efforts d'atténuation de la crise, aussi bien de la part de Nicolas Sarkozy que de la part de Cavaco Silva et de Traian Băsescu. On y retrouve des volitifs, des déontiques et des auxiliaires modaux (du devoir notamment), ce qui montre bien à quel point l'expression de l'obligation occupe une place importante dans les discours de 2010 :

NS : Mes chers compatriotes, même si les épreuves ne sont pas terminées, 2010 sera une année de renouveau. Les efforts que nous faisons depuis deux ans et demi vont porter leurs fruits. Dans ce moment si crucial nous devons rester unis comme nous avons su l'être au plus fort de la crise. C'est cette unité qui nous a permis de prendre l'initiative d'entraîner les autres. Les idées que la France défend vont pouvoir s'imposer dans la recherche d'un nouvel ordre mondial : plus d'équilibre, plus de régulation, davantage de justice et de paix. Ces idées nous imposent un devoir d'exemplarité. Respectons-nous les uns les autres, faisons l'effort de nous comprendre, évitons les mots et les attitudes qui blessent. Soyons capables de débattre sans nous déchirer, sans nous insulter, sans nous désunir.

CS : Neste primeiro dia do ano, importa reafirmar o valor da esperança. Repito aos Portugueses o que lhes disse há precisamente um ano : não tenham medo. Possuímos uma longa História de que nos orgulhamos, porque no passado não tivemos medo. E aqui estamos hoje, um Estado democrático que faz parte de uma Europa Unida. Aqui estamos hoje, em 2010, porque acreditámos em nós próprios e num destino chamado futuro. Em nome desse futuro, temos de continuar a lutar. $O$ combate que travamos por Portugal é feito em nosso nome e em nome dos nossos filhos. ${ }^{17}$

TS : Nu avem de ce să nu fim mândri de ţara noastră. Istoria ţării, cultura, tradiţiile noastre sunt cele care îi recomandă pe români ca una din cele mai puternice naţiuni ale Uniunii Europene. Din acest motiv, cred că fiecare trebuie să avem în minte că dragostea de ţară înseamnă patriotism. lar, patriotismul este parte a solidarităţii noastre cu ţara în care trăim, cu ţara pe care o iubim. Peste numai două minute, vom intra în anul 2010. Va fi un an în care vom scăpa de criză, va fi un an în care optimismul va fi cel care ne va anima să depăşim momentul pe care l-am trăit în anul 2009. ${ }^{18}$

17. «En ce premier jour de l'année, il est important de réaffirmer la valeur de l'espoir. Je répète aux Portugais ce que je leur ai déjà dit il y a un an précisément : n’ayez pas peur. Nous avons une longue histoire dont nous sommes fiers parce que dans le passé nous n'avons pas eu peur. Et nous voici, aujourd'hui, un État démocratique qui fait partie d'une Europe unie. Nous voici aujourd'hui, en 2010, parce que nous avons cru en nous-mêmes et dans un destin appelé avenir. Au nom de cet avenir, nous devons continuer à lutter. La bataille que nous menons pour le Portugal se fait en notre nom et au nom de nos enfants. "

18. «Nous n'avons aucune raison de ne pas être fiers de notre pays. L'histoire du pays, la culture, nos traditions sont celles qui recommandent les Roumains comme l'une des nations les plus puissantes de l'Union européenne. Pour cette raison, je crois que chacun doit avoir à l'esprit que l'amour pour la patrie signifie le patriotisme. Et le patriotisme est une partie de notre solidarité avec le pays dans lequel nous vivons, avec le pays que nous aimons. Dans deux minutes, 
La présence de verbes d'action, de jugements et de modalités appréciatives associés à la première personne du pluriel produit un effet d'effacement de la personnalité des trois présidents derrière leur fonction. Il y a par conséquent une reconnaissance explicite des dysfonctionnements évoqués, mais cette reconnaissance est accompagnée aussitôt d'une mise en équilibre qui tend à relativiser les choses. Les vœux en tant que formules rituelles sont peu présents dans les allocutions de 2009-2010, car la politique extérieure et les crises à l'intérieur du pays occupent une place importante dans les discours des trois présidents. Les séquences suivent toutes les trois la même logique : une argumentation centrée sur le rassemblement et l'unité du peuple, suivie par l'appel solennel à la confiance. Pour ce qui est des formes verbales, le renvoi au présent et au passé contribue à l'expression du bilan des actions présidentielles, lequel est également ouvert sur l'avenir (emploi de l'impératif et du futur de l'indicatif). Après les constats sur la crise, l'accent est mis sur ce qui fait la force de ces pays, c'est-à-dire l'unité et le rassemblement.

En nous situant dans la représentation trimorphique de la zone d'éloignement qui correspond aux séquences finales de notre corpus, nous observons une approche commune de la crise qui se traduit par des énoncés orientés vers l'avenir et porteurs d'espérance. Ainsi, la notion de confiance - élément essentiel des vœux dans les trois cas - se construit au niveau textuel à l'aide des verbes au futur. La crise économique est là, mais le Portugal, la France et la Roumanie sont de "grands pays". L'évocation de cette grandeur et des épreuves passées est commune aux trois discours.

Les séquences finales en français comportent des formules rituelles caractérisées par un haut degré de figement; il s'agit d'un souhait adressé à la République et d'un souhait pour la France.

NS, 2008-2009: Vive la République et vive la France!

NS, 2009-2010: Mes chers compatriotes, vive la République et vive la France.

Dans ses vœux, Cavaco Silva utilise son slogan de campagne - «Acredito em Portugal »-dans les séquences de clôture, ce qui s'explique par l'approche des élections présidentielles de 2011 où il brigue un second mandat :

CS, 2008-2009: Acredito num futuro melhor e mais justo para Portugal, porque acredito na vontade e no querer do nosso povo. Para todos, Bom Ano de 2009. ${ }^{19}$

CS, 2009-2010 : Eu acredito em Portugal. Por isso, continuarei a lutar pelo futuro desta nossa terra. No meio de tantas incertezas, os Portugueses podem ter uma cer-

nous entrerons en 2010. Ce sera une année dans laquelle nous échapperons à la crise, ce sera une année au cours de laquelle l'optimisme nous animera pour dépasser la période que nous avons vécue en 2009."

19. "Je crois dans un futur meilleur et plus juste pour le Portugal, parce que je crois dans la volonté et l'aspiration de notre peuple. Pour tous, bonne année 2009.» 
teza : pela minha parte, não desistirei e nunca me afastarei dos meus deveres e dos meus compromissos. A todos, um Bom Ano de 2010 !20

Quant aux deux discours du président roumain, la séquence verbale finale est moins figée par rapport aux séquences de clôture en portugais et en français.

TB, 2008-2009 : Şi pentru că ultimele clipe ale anului care trec le trecem împreună, daţi-mi voie, de aici, de la Braşov, să vă doresc tuturor din tot sufletul, cu toată dragostea, «La mulţi ani! » şi «Să trăiţi bine ! »21

TB, 2009-2010 : Permiteţi-mi dragi români să închei urându-vă multă sănătate, bucurie, fericire în familii şi, cel mai important lucru, solidaritate. Solidaritatea cu noi înşine şi cu obiectivele noastre. Vă doresc la toţi, La mulţi ani! La mulţi ani, România! La mulţi ani, români! La mulţi ani, ţară dragă !22

Cette manière de clore ses vœux s'explique par des circonstances politiques bien précises. D’une part, le soin déjà mentionné d'éviter tout rapprochement avec les discours de Nicolae Ceauşescu, qui finissait toujours ses allocutions par la formule «Vive la République socialiste de Roumanie»; d'autre part, tout comme dans le cas du président portugais, le désir de Traian Băsescu de rappeler aux Roumains son slogan de campagne pour l'année électorale à venir : «Să trăiţi bine!» 23

Àtravers les marques énonciatives analysées, lesvœux présidentiels construisent une représentation du locuteur et de son destinataire. Ces profils énonciatifs très diversifiés fournissent des indices sur la conception de la fonction présidentielle que se font les locuteurs, sur la vision qu'ils ont de leur peuple mais également sur la façon dont ils conçoivent la crise et les messages de vœux. Notre analyse a essayé de montrer que les formes d'adresse reflètent la personnalisation du discours politique et sont porteuses de valeurs. Ainsi, les présidents s'adressent à leurs allocutaires au sein d'une relation d'hyperonymie/hyponymie qui se traduit par l'emploi de classes de destinataires (à travers des termes relationnels: Mes chers compatriotes ou bien des formes allocutives génériques : Français,

20. «Je fais confiance au Portugal. Par conséquent, je vais continuer à me battre pour l'avenir de notre pays. Au milieu de tant d'incertitudes, les Portugais peuvent avoir une certitude : pour ma part, je n'abandonnerai pas et je n'oublierai jamais mes devoirs et mes engagements. À tous, bonne année 2010 !»

21. «Et parce que nous passons ensemble les derniers moments de l'année qui s'écoule permettez-moi d'ici, de Brasov, de vous souhaiter à tous de toute mon âme, de tout mon cœur : "Bonne année!" et "Vivez bien!” "

22. "Permettez-moi chers Roumains de finir en vous souhaitant bonne santé, des joies et du bonheur dans vos familles et la chose la plus importante, de la solidarité. La solidarité avec nousmêmes et avec nos objectifs. Je vous souhaite à tous : Bonne année! Bonne année, Roumanie! Bonne année, Roumains! Bonne année, cher pays!»

23. Le slogan de campagne est construit à l'aide du salut spécifique au langage militaire : «Să trăiţi » (voir à ce propos l'analyse de Zafiu 2007, p. 57) et l'adverbe bien qui modalise axiologiquement le syntagme verbal. 
Portugais, Roumains) et des sous-classes (agriculteurs, jeunes, petits commerçants, militaires, etc.). Ce sont ces formes nominales d'adresse fonctionnant comme relationèmes (Kerbrat-Orecchioni, 1992, p. 37) qui permettent d'inscrire les auditeurs dans une classe dont les membres ont une caractéristique commune (Détrie, 2006, p. 63).

Nous retrouvons également des formes pronominales d'adresse qui contribuent à la construction de l'ethos présidentiel. Ainsi, le président portugais construit plutôt un discours de la distance, en utilisant la $3^{\mathrm{e}}$ personne du pluriel et en marquant une relation « je vs les Portugais ». Le rapprochement énonciatif est produit notamment par l'usage des marqueurs de temps. Le président Cavaco Silva centre ainsi son discours sur lui-même - «je l'ai déjà dit » -, construisant de façon subtile un jeu à la fois monologal et dialogal. Sous l'apparence d'un locuteur qui revient sur son propre discours, c'est le prolongement du dialogue entre le président et ses concitoyens qui est suggéré. Le discours de Cavaco Silva se caractérise donc par une prise de distance énonciative produite par l'usage peu fréquent des marques de première personne, la prise en charge étant assurée par une troisième personne dont le référent principal est le Portugal et les Portugais. Il incarne l'image d'un président dont les valeurs principales sont la raison, le bon sens et la vérité.

À l'opposé, Traian Băsescu multiplie les marques énonciatives à l'intention des Roumains tout en personnalisant son discours par l'usage fréquent de la première personne du singulier. Les déclinaisons de toutes les catégories de Roumains, l'omniprésence des marques de l'énonciation ("j'adresse», "je vous souhaite ») et des marques affectives ("de tout mon cœur», «je suis heureux») ancrent les discours du président roumain dans un hic et nunc qui a pour but d'ignorer et de nier les effets de la crise. L'allocution de Traian Băsescu met en scène l'image d'un président qui instaure une relation plutôt amicale et familière avec les Roumains, qui cherche à imposer le parlervrai à tout prix.

Nicolas Sarkozy, quant à lui, privilégie les marques de première personne du singulier dans son discours de 2009 , tout en utilisant des marques de la première personne du pluriel en 2010. Nous notons la place du je dans les vœux du président français qui s'exprime plus que les deux autres présidents à la première personne : on trouve notamment des possessifs présents dans les formules d'adresse et le pronom personnel dans des emplois épistémiques («je sais », "je pense »). Cependant, il s'agit en même temps d'un discours tourné vers l'action, qui se construit à l'aide de verbes volitifs et de modalités appréciatives associés à la première personne du singulier. De cette façon, le président s'engage personnellement et lance un appel direct aux Français. II s'agit d'un discours du « vouloir faire», "performatif», selon Damon Mayaffre (2012), incarné par un personnage soucieux également de «dire la vérité aux Français».

De plus, si nous nous rapportons à la représentation trimorphique de Bernard Pottier, nous constatons que la plupart des allocutions présidentielles 
conçoivent l'événement «crise» de la même façon : 1) une zone d'approche visant à évoquer les actions des trois présidents et l'année écoulée, décrite dysphoriquement ; 2) une zone de contact, « dire la vérité », construite grâce à l'emploi du présent, donnant surtout un effet descriptif de la crise ; 3) une zone d'éloignement créée grâce à l'emploi des verbes au futur, conjugués à la première personne du pluriel et suggérant une évocation optimiste de l'avenir. Le futur a une dimension prédictive, renforçant ainsi l'ethos présidentiel et montrant les chefs d'État sous des traits rassurants.

\section{Références}

Amossy Ruth, 2000, L'argumentation dans le discours. Discours politique, littérature d'idées, fiction, Paris, Nathan Université.

Araújo Carreira Maria Helena, 1997, Modalisation linguistique en situation d'interlocution. Proxémique verbale et modalités en portugais, Louvain-Paris, Peeters.

- 2005, "La construction discursive de la désignation de l'autre et de soi-même. L'exemple du discours d'opinion sur les élections (portugais) et du discours électoral (français) », Texte et discours. Catégories pour l'analyse, J.-M. Adam, J.-B. Grize et M. A. Bouacha éd., Dijon, PUD, p.145-162.

— éd., 2008, "Mignonne, allons voir si la rose... » Termes d'adresse et modalités énonciatives dans les langues romanes », Travaux et documents, n 40 , Saint-Denis, Université Paris 8.

Ciolac Marina, 2010, «Vœux présidentiels télédiffusés dans deux États de l'Union européenne», Signes, discours et sociétés, n⿳05. Communication et discours politiques. Actualités et perspectives, 4 juin 2010, en ligne : http ://revue-signes. info/ document. php? id=1622. ISSN 1308-8378 (consulté le 6 décembre 2012).

DÉTRIE Catherine, 2006, De la non-personne à la personne. L'apostrophe nominale, Paris, CNRS.

Kerbrat-Orecchioni Catherine, 1992, Les interactions verbales, t.II, Paris, Armand Colin.

- 1990, L'énonciation. De la subjectivité dans le langage, Paris, Armand Colin.

LEBLANC Jean-Marc, 2003, "Les messages de vœux des présidents de la Cinquième République. L'ethos, la diachronie, deux facteurs de la variation lexicométrique », Lexicométrica, no 4 , en ligne : http://lexicometrica.univ-paris3.fr/article/ numero4/2.pdf (consulté le 6 décembre 2012).

- 2005, "Les vœux présidentiels sous la Cinquième République (1959-2001). Recherches et expérimentations lexicométriques à propos de l'ethos dans un genre discursif rituel», thèse de doctorat en sciences du langage, sous la direction de Pierre Fiala, Université Paris 12 Val-de-Marne, en ligne : http://leblanc.jeanmarc. free.fr/These/ThseJM_Leblanc.pdf (consulté le 6 décembre 2012).

LEB LANC Jean-Marc, MARTINEZ William, 2005, «Positionnements énonciatifs dans les vœux présidentiels sous la Cinquième République. Analyse des marques personnelles par 
les méthodes de cooccurrence», Corpus, $\mathrm{n}^{\circ} 4$, décembre 2005, en ligne : http://corpus. revues.org/index347.html (consulté le 6 décembre 2012).

LEBLANC Jean-Marc, FIALA Pierre, 2004, «Autour du Je présidentiel», JADT'04 : Le poids des mots, Actes des VIle Journées internationales d'analyse statistique des données textuelles, vol. Il, Louvain-la-Neuve, Presses universitaires de Louvain (UCL), p. 716-725.

MAYAfrRe Damon, 2012, Nicolas Sarkozy. Mesure et démesure du discours, 2007-2012, Paris, Presses de Sciences Po.

PotTier Bernard, 1992, Sémantique générale, Paris, PUF.

ZAfIU Rodica, 2007, Limbaj şi politică, Bucarest, Editura Universităţii din Bucureşti. 